\title{
Energy savings approach to optimal location of EV charging stations in microgrids
}

\author{
Vishnu Suresh $^{1}$, Przemyslaw Janik ${ }^{1}$, Dominika Kaczorowska ${ }^{1}$ \\ ${ }^{1}$ Faculty of Electrical Engineering \\ Wroclaw University of Science and Technology \\ Wybrzeże Stanisława Wyspiańskiego 27, 50-370 Wrocław (Poland) \\ Phone/Fax number: +48 7132029 05, e-mail: vishnu.suresh@pwr.edu.pl , przemyslaw.janik@ pwr.edu.pl
}

\begin{abstract}
This paper presents an analytical approach to finding an optimal location for an EV charging station based on energy savings in a local microgrid. The analysis is carried out on days obtained by clustering yearly load data and by running an energy management system that runs on MATLAB interior point method. The microgrid is composed of both renewable and non-renewable energy sources. The charging station is equipped with a controlled charging feature and this study considers 2 EV charging strategies out of which the one benefitting the power system is adopted.
\end{abstract}

Key words. microgrids, EV charging station optimal location, interior point method, renewable energy, energy management systems

\section{Introduction}

At present there is a growing demand for the society to be sustainable and eco-friendly. This has resulted in calls for transformation of both the energy and transportation sectors $[1,2]$. EVs are seen as a potential alternative to fossil fuelled driven road transportation vehicles. In fact, their adoption has been on the rise and they cover about one-third of the market share in Norway and $6.4 \%$ in the Netherlands. Numerous other countries intend to follow this direction and are aiming for $100 \% \mathrm{EV}$ adoption sooner or later[1].

While the EVs represent a sustainable, green and low carbon mode of transportation its adoption faces numerous challenges which include limited range of travel, long waiting times for charging, unprepared distribution networks and lack of adequate Electrical Vehicle Charging System (EVCS) [3-5]. Amongst the above-mentioned challenges, it is also argued that EVs powered by energy from eco-friendly sources are more sustainable than those powered by fossil fuels.

In the literature numerous approaches of locating EV charging stations in distribution grids are present, some of which are discussed below. An investigation where the results were verified by means of a real case study is presented in [4]. In this investigation the problem is designed as a Mixed Integer Linear Programming (MILP) problem which considers the Geographic Information System (GIS) to find the right location for an EVCS. It also considers traffic behaviour and land-use classifications in its calculation. The objective function used is maximization of the return on investments. The case study is implemented in Vasteras, Sweden. Another comprehensive study where the placement of the EVCS is determined by taking into account numerous factors is shown in [1]. Here, the type of charger used is taken into account and a right combination of level 1, level 2 and level 3 chargers is determined by keeping in mind the capacity of the distribution grid to handle sudden increases in the load demand by EVCSs. Apart from this it also considers the effect of the power produced by solar panels and the objective function minimizes the installation cost along with the cost associated with power losses. Particle Swarm Optimization (PSO) is chosen as the optimization algorithm and the models are established on MATLAB and OpenDSS. The approach is validated on a real distribution grid in Pakistan. A study of EVCS dynamics in a residential neighbourhood is presented in [3]. The study implements co-ordinated charging strategies in order to reduce the costs associated with charging the EVs and protect the distribution transformer from overloading. The study is implemented on four days that typically represent most of the days in a year including the holiday period. Genetic Algorithm (GA) is used to solve the mathematical model created. A cross-entropy approach to planning the location and size of the EVCS is presented in [6]. The study utilizes a multi-objective function which minimizes the power losses and voltage fluctuations in the power system. It also attempts to maximise the flow of traffic. The results are validated by means of a case study using a 33-node distribution system and a 25-node traffic network system. A data mining approach to finding an optimal location for EVCSs in presented in [7]. The investigation has been made specifically for the city of Ankara, Turkey where the study has considered the road map of the city that they downloaded using Mapbox from satellite images using spectral clustering. The study considers both the average range of typical EVs and the total number of EVs on the road. Finally, the location is determined using spectral clustering and Gaussian Mixture Model. It has been presented as a novel study for the region under consideration. More studies in this direction involving microgrids, renewable energies and charging stations can be found in [8-13] 
While numerous approaches have been presented above there are still many considerations by which an optimal location of EVCSs can be obtained. Keeping in line with the sustainability motives of the present, this study presents the optimal location of an EVCS in a microgrid consisting of a significant amount of energy produced from solar panels and li-ion storage device.

The contributions of the article are as follows:

- Identification of an optimal location for the EVCS in the local microgrid.

- Co-ordinated charging explored in terms of controlled and uncontrolled charging.

- Clustering to identify days representing the whole year in order to calculate energy savings.

The rest of the paper is presented as follows: section 2 describes the microgrid layout of the study, sections 3 describes the mathematical model and the energy management procedure. Section 4 describes the results and section 5 enumerates the conclusions of the study.

\section{Microgrid layout}

The layout of the microgrid can be seen in Fig. 1. G1 is the main grid. There are in total 6 generators in the microgrid wherein 3 generators represent power from solar PV modules. They are generators G3, G4 and G5 located on nodes 2,3 and 4 with a peak power capacity of $6.5 \mathrm{~kW}, 9 \mathrm{k}$ and $12.5 \mathrm{~kW}$ respectively. There are $2 \mathrm{DG}$ units located in nodes 2 and 5 with a peak power capacity of $5.2 \mathrm{~kW}$ each. Finally, the storage device with a capacity of $9.8 \mathrm{kWh}$ is located at node 5 and is designated as G7/L5. The data of the PV modules is taken from panels located on the roof of the faculty at Wroclaw University of Science and Technology. The data is multiplied suitably by constants to represent G3, G4 and G5.

The load data also available with the university is multiplied by suitable constants to represent different loads. There are in total 5 loads within the microgrid. Load 1, Load 2, Load 3 and Load 4 are located on nodes 2, 3, 4 and 5 with peak loads of $14.5 \mathrm{~kW}, 7.5 \mathrm{~kW}, 11 \mathrm{~kW}$ and $8.5 \mathrm{~kW}$ respectively. The final load L5 is the storage device when it is charging.

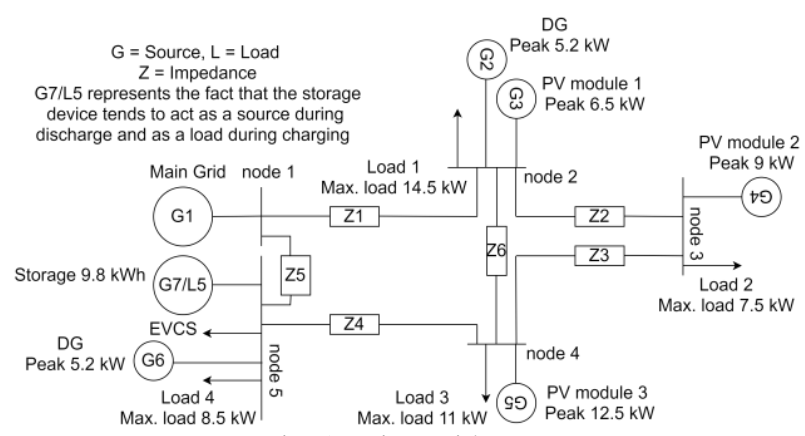

Fig. 1. Microgrid Layout

The lines connecting different nodes are represented by $\mathbf{Z 1}$, $\mathrm{Z} 2, \mathrm{Z3}, \mathrm{Z4}, \mathrm{Z} 5$ and Z6. The cables used are IEC standard 60502-1:2004 lines. It is made from aluminium and has an XLPE insulation.
Table I. - Cable Data

\begin{tabular}{|c|c|c|c|}
\hline From & To & Distance $(\mathrm{m})$ & $r+j x(\mathrm{ohm}) * 10^{-1}$ \\
\hline node 1 & node 2 & 220 & $0.557+0.180 \mathrm{j}$ \\
\hline node 2 & node 3 & 130 & $0.329+0.106 \mathrm{j}$ \\
\hline node 3 & node 4 & 145 & $0.367+0.118 \mathrm{j}$ \\
\hline node 4 & node 5 & 180 & $0.455+0.147 \mathrm{j}$ \\
\hline node 5 & node 1 & 170 & $0.430+0.139 \mathrm{j}$ \\
\hline node 2 & node 4 & 250 & $0.633+0.204 \mathrm{j}$ \\
\hline
\end{tabular}

The EVCS currently located at node 5 of the microgrid has in total 5 Tesla level 2 chargers. The total power delivery per hour of the chargers lie between $3.7 \mathrm{~kW}$ and $17.2 \mathrm{~kW}$. Their optimal location in the microgrid will be determined at the end of the paper.

\section{Mathematical foundation and Energy management}

\section{A. Mathematical Foundation}

Firstly, the objective function of the Energy Management System (EMS), equality and inequality constraints are defined after which the mathematical model of the EVCS is defined. The objective function is shown in (1).

$$
\min \left(P_{G}\right)=P_{d}+P_{l}-P_{m g}
$$

Subject to equality constraints

$$
\begin{gathered}
\sum P_{g i}-\sum P_{d i}-P_{L}=0 \forall i \epsilon \text { No. of nodes } \\
\sum Q_{g i}-\sum Q_{d i}-Q_{L}=0 \forall i \epsilon \text { No. of nodes }
\end{gathered}
$$

Subject to inequality constraints

$$
\begin{gathered}
P_{g i(\text { minimum })} \leq P_{g i} \leq P_{g i(\text { maximum })} \\
Q_{g i(\text { minimum })} \leq Q_{g i} \leq Q_{g i(\text { maximum })} \\
V_{i(\text { minimum })} \leq V_{i} \leq V_{i(\text { maximum })} \\
\delta_{i(\text { minimum })} \leq \delta_{i} \leq \delta_{i(\text { maximum })}
\end{gathered}
$$

The objective function of the study is to minimize the import of power from the main grid $\left(\mathrm{P}_{\mathrm{G}}\right) . \mathrm{P}_{\mathrm{d}}$ is total power demand in the microgrid, $\mathrm{P}_{1}$ represents the line losses whereas $\mathrm{P}_{\mathrm{mg}}$ is the power produced in the microgrid. This particular objective function was chosen in order to study the dynamics of the system when all sources of energy in the microgrid are used at their maximum capacity. This is important because the university eventually plans to move to a microgrid that is stand-alone and not grid connected. (2) and (3) are the equations required to carry out power balancing in the microgrid where $\mathrm{P}_{\mathrm{gi}}$ and $\mathrm{Q}_{\mathrm{gi}}$ represent active power and reactive power that is generated at node $\mathrm{i}, \mathrm{P}_{\mathrm{di}}$ and $\mathrm{Q}_{\mathrm{di}}$ represent active power and reactive power demand at node i, $P_{L}$ and $Q_{L}$ are active and reactive power losses. The voltage value at node $\mathrm{i}$ is represented by $\mathrm{V}_{\mathrm{i}} . \delta_{i}$ is the voltage angle at node $i$.

The mathematical model concerning the EVCS is as follows:

$$
N_{E V}^{t} \leq N_{c}^{t}
$$




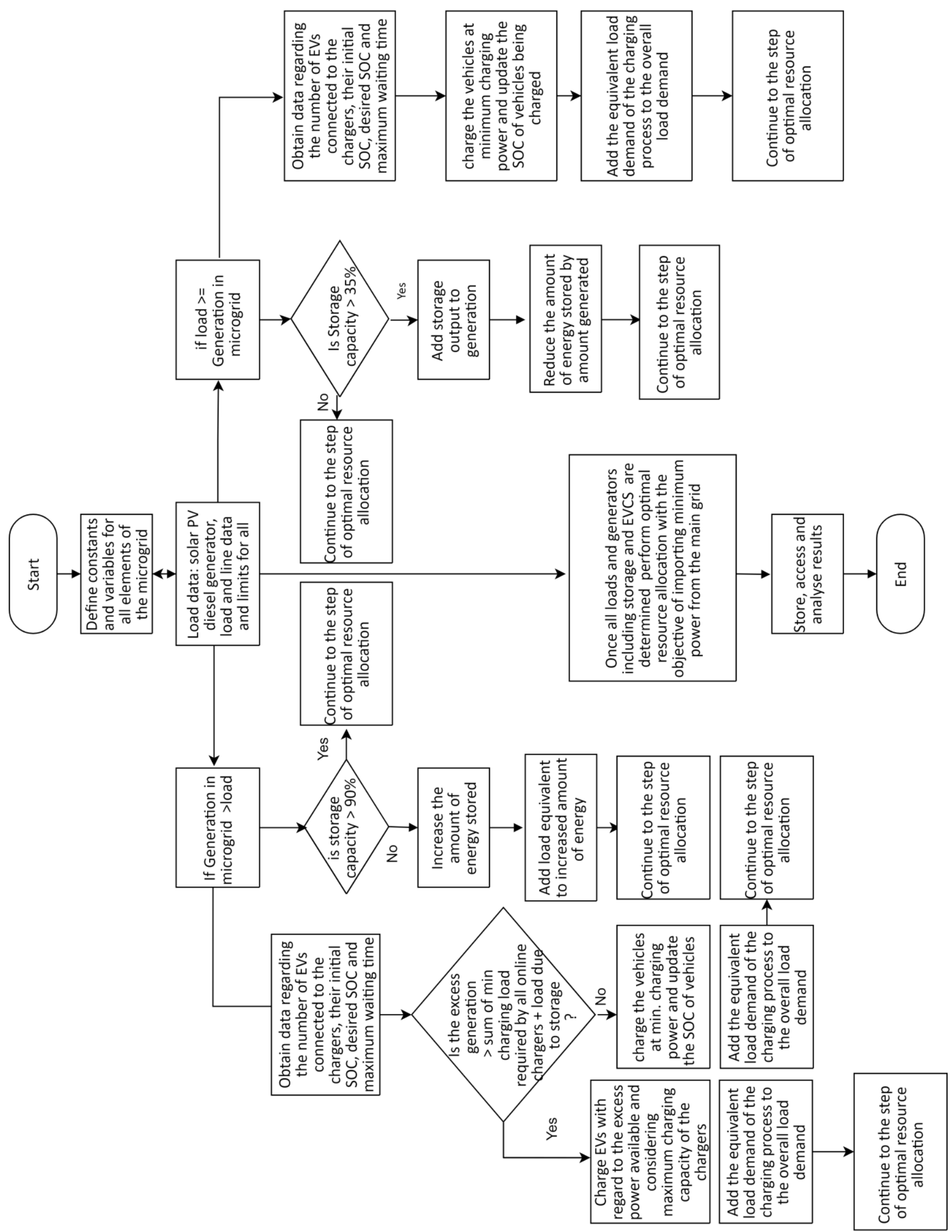

Fig. 2 Energy management 


$$
\begin{gathered}
P_{c m i n i m u m} \leq P_{c t} \leq P_{c m a x i m u m} \\
S O C_{i}^{\text {initial }} \leq S O C_{i} \leq S O C_{i}^{\text {desired }}, i=1,2 \ldots \ldots N_{E V} \\
a d d_{S O C}=\Delta t * \sum_{k=1}^{N} P_{c t} \\
T_{c}^{i} \leq \min \left(T_{\text {max }}^{i}, T_{d e s}^{i}\right)
\end{gathered}
$$

(8) ensures that the number of EVs connected $\mathrm{N}_{\mathrm{EV}}^{\mathrm{t}}$ at every time step $t$ are equal to less than the total number of chargers $\mathrm{N}_{\mathrm{c}}^{\mathrm{t}}$. (9) is a constraint that ensures that the power delivered $\mathrm{P}_{\mathrm{ct}}$ by every charger is within its minimum and maximum rated power delivery. (10) ensures that for every vehicle i, the State of Charge $\left(\mathrm{SOC}_{\mathrm{i}}\right)$ is within its value during its arrival $\left(\mathrm{SOC}_{\mathrm{i}}^{\text {initial }}\right)$ and the desired SOC value $\left(\mathrm{SOC}_{\text {desired }}^{\mathrm{i}}\right)$ input by the user. The increment of the SOC for every vehicle is denoted by $\operatorname{add}_{\mathrm{SOC}}, \Delta t$ is the length of every time step and $\mathrm{P}_{\mathrm{ct}}$ is the power delivered. (12) represents the fact that the time of departure for every vehicle $\mathrm{i}\left(\mathrm{T}_{\mathrm{c}}^{\mathrm{i}}\right)$ is the lowest of the choice between the maximum waiting time input by the user $\mathrm{T}_{\text {maximum }}^{\mathrm{i}}$ and time taken to reach the desired SOC value $\mathrm{T}_{\mathrm{des}}^{\mathrm{i}}$ input by the user.

\section{A. Energy management}

The energy management strategy is shown in Fig. 2 and is explained below.

The process begins with the identification and characterisation of the microgrid and all its elements. This includes the electrical parameters and limits of all generators, loads and cables.

Once this step is complete, the energy management happens under two distinct conditions. The first condition is triggered when the generation in the microgrid is greater than the load and the second condition is triggered when the load is greater than the generation in the microgrid. Under both conditions the EMS views the main grid as the slack generator which is capable of supplying and receiving active and reactive power according to the needs of the system.

Under the first condition first the SOC of the storage system is determined. If it is greater than $90 \%$, the storage device is not charged, if it is less than $90 \%$ charging takes place. Apart from this for the EVCS it is checked whether the excess power minus the power consumed by the storage device is greater than the combined demand of all connected chargers when they deliver power at their minimum rated power. If it is greater the optimization algorithm determines the amount of power delivered by the chargers, if not, all chargers withdraw power at their minimum rated power delivery. Under the second condition again the SOC of the storage system is determined, if it is less than $35 \%$ no discharge takes place, if it is greater than $35 \%$ the storage system starts discharging. For the EVCS under this condition all chargers deliver power at their minimum rated power delivery.

\section{Results}

In order to have a wholesome picture of the dynamics of the microgrid in an entire year. The load data was clustered using the k-means clustering algorithm. This resulted in a total of 3 clusters around which all days in a year can be classified on. The k-means algorithm is simple to implement and is explained in detail in [12]. A day close to the centroid of each cluster was chosen and the renewable energy produced on that day along with the load were obtained. The figures corresponding to them are shown below.
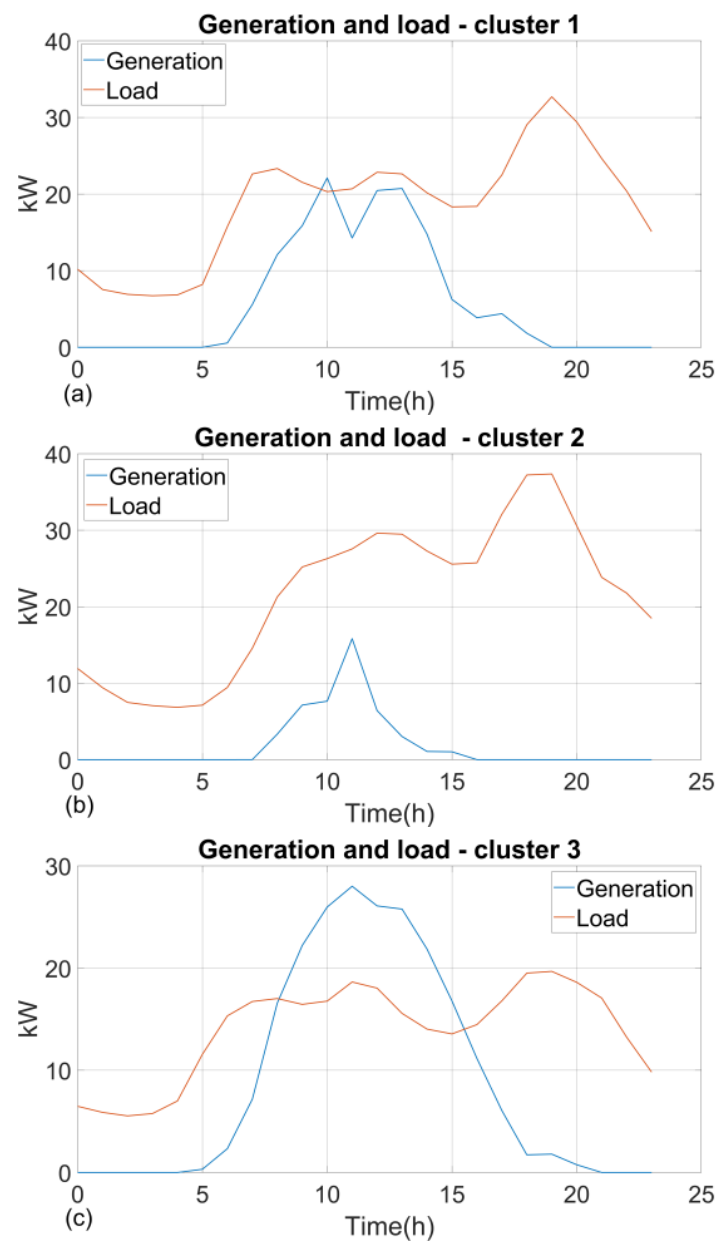

Fig. 3. Days representing an entire year (a) cluster -1 (spring, autumn) (b) cluster -2 (winter) (c) cluster -3 (summer)

From Fig. 3 it can be inferred that cluster -1 is representative of both the spring and autumn when both the power generated withing the microgrid and the load demand are somewhere in between their peak and minimum values. Cluster -2 representing the winter is a time period during which the power generated in the microgrid remains very low and the load very high. This is because of low availability of sunlight and the harsh winter during which heating devices are regularly used. During this period reliance on the external grid is high. Cluster -3 is typical for the summer wherein the power generated is very high due to high availability of sunlight. Moreover, the summer is quite mild which keeps the load demand quite low. The reliance on the grid during this time period is lower when compared to other time periods.

Fig. 4 shows the load characteristic for the day from cluster 1 when controlled charging and uncontrolled charging are applied separately. It can be seen that when 
the power delivery from the chargers is not controlled, the load characteristic is affected significantly and is very stochastic with high peaks and sharp falls. Moreover, the frequency of such peaks and falls are high indicating that the power system is stressed during such an operation. On the other hand, when the charging is controlled the load curve is smooth which ensures healthy operation of the power system.

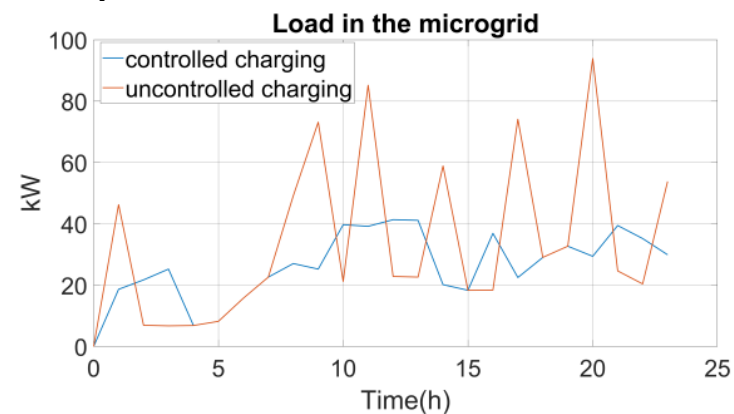

Fig. 4. Load characteristic under controlled and uncontrolled charging

Fig. 5 shows the power exchange characteristic during the same time period and it can be seen that much of the power demand due to the uncontrolled charging is met by importing power from the external grid. This can be noticed by the peaks in both Fig. 4 and Fig. 5 which are similar to one another.

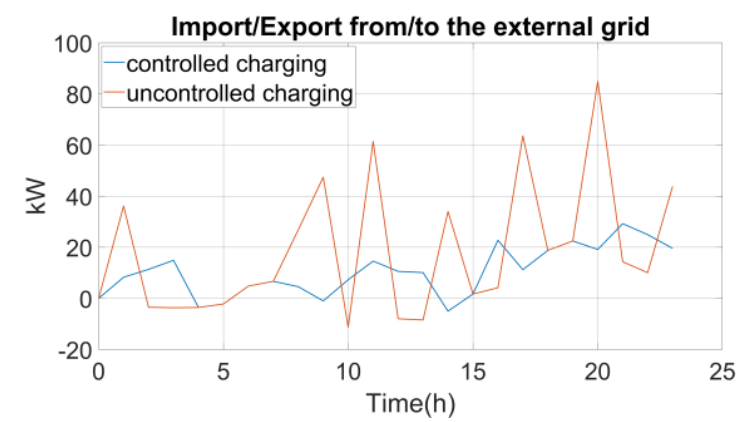

Fig. 5. Power exchange characteristic under controlled and uncontrolled charging

It is possible to track the SOC of the incoming EVs during their charging process . Fig. 6 shows the SOCs of different EVs being tracked at chargers 1 and 2 of the EVCS during the controlled charging scenario.

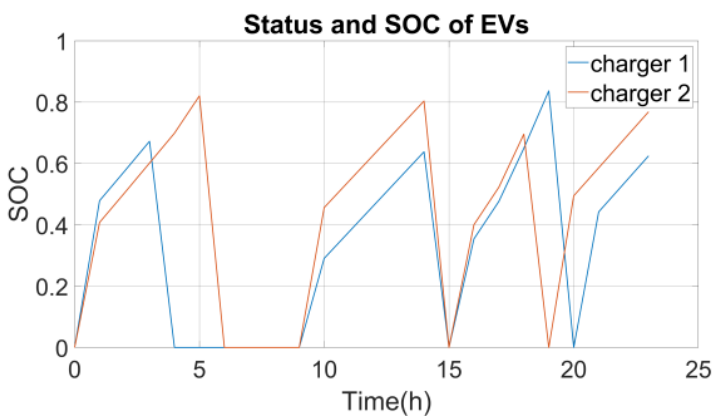

Fig. 6. SOC tracking for chargers 1 and 2 in the EVCS under controlled charging

It can be seen from the figure that in both chargers 1 and 2 , 3 EVs were charged up to their desired inputs (maximum waiting time or time taken to reach desired SOC).
In order to determine the optimal location of the EVCS in the microgrid, an analysis has to made on the total amount of energy that would be imported when it is placed on each node considering a day from every cluster. Table 2 presents the information for this analysis.

Table II. Total net - import of energy based on different location of the EVCS on different days.

\begin{tabular}{|c|c|c|c|}
\hline Node & $\begin{array}{c}\text { Cluster 1 } \\
\text { Net-import } \\
(\mathbf{k W h})\end{array}$ & $\begin{array}{c}\text { Cluster 2 } \\
\text { Net-import } \\
\mathbf{( k W h )}\end{array}$ & $\begin{array}{c}\text { Cluster 3 } \\
\text { Net-import } \\
\mathbf{( k W h )}\end{array}$ \\
\hline 2 & 251.20 & 493.68 & 89.58 \\
\hline 3 & 251.53 & 494.43 & 89.86 \\
\hline 4 & 251.37 & 494.11 & 89.70 \\
\hline 5 & 251.04 & 492.97 & 89.61 \\
\hline
\end{tabular}

From table 2, it can be seen that the location of the EVCS does have an impact on the net energy imported from the grid even though it is small. This difference is especially seen during the wintertime when much of the energy used is imported from the main grid. It is so because node 5 is the closest to the main grid amongst all nodes. During other times in the year the two closest nodes compete with each other in terms of energy savings (nodes 2 and 5). Nodes 3 and 4 are still not competitive since the power imported from the main grid is significant on all days but it can be predicted that if the self-reliability of the microgrid is improved in terms of generated power these nodes could become competitive and be good locations for the EVCS. At present given the current layout of the microgrid the optimal location for the EVCS is at node 5. The node closest to the main grid.

\section{Conclusions}

This paper presented an approach to optimally locating an EVCS in a local microgrid. The final decision on the location was based on an analysis of the energy savings observed by placing the EVCS on different nodes in the microgrid for days representing the whole year. These representative days were obtained by using k-means clustering that resulted in the formation of 3 clusters. One representing winter where the load is high and energy produced in microgrid is low, another representing the summer where the load is low and the power produced in the microgrid is high and finally a cluster representing autumn and spring where both the load and the power generated are in between the extremes. An energy management strategy was devised in order to maximise the utilisation of energy sources in microgrid and discourage the import of power in the main grid. Two charging strategies were explored which are the controlled charging and the uncontrolled charging. It was shown that the uncontrolled charging strained the functioning of the microgrid. Moreover, it was also shown that uncontrolled charging significantly increased the reliance on the main grid. This work can be extended in many ways. The microgrid can be made more diverse with integration of additional renewable sources of energy, additional sources 
of different renewable energy sources, more charging scenarios can be explored, the traffic patterns of the city of Wroclaw can be studied, range of EVs could be taken into consideration and other objective functions could be considered for the EMS.

\section{References}

[1] M.Z. Zeb, K. Imran, A. Khattak, A.K. Janjua, A. Pal, M. Nadeem, J. Zhang, S. Khan, Optimal Placement of Electric Vehicle Charging Stations in the Active Distribution Network, IEEE Access. 8 (2020) 6812468134. https://doi.org/10.1109/ACCESS.2020.2984127.

[2] H.C. Liu, M. Yang, M. Zhou, G. Tian, An Integrated Multi-Criteria Decision Making Approach to Location Planning of Electric Vehicle Charging Stations, IEEE Trans. Intell. Transp. Syst. 20 (2019) 362-373. https://doi.org/10.1109/TITS.2018.2815680.

[3] L. Gong, W. Cao, K. Liu, J. Zhao, Optimal charging strategy for electric vehicles in residential charging station under dynamic spike pricing policy, Sustain. $\begin{array}{lllll}\text { Cities } & \text { Soc. } & 63 & \text { (2020) } & 102474 .\end{array}$ https://doi.org/10.1016/j.scs.2020.102474.

[4] C. Bian, H. Li, F. Wallin, A. Avelin, L. Lin, Z. Yu, Finding the optimal location for public charging stations - A GIS-based MILP approach, Energy Procedia. 158 (2019) $6582-6588$. https://doi.org/10.1016/j.egypro.2019.01.071.

[5] J. He, H. Yang, T.Q. Tang, H.J. Huang, An optimal charging station location model with the consideration of electric vehicle's driving range, Transp. Res. Part C Emerg. Technol. $86 \quad$ (2018) 641-654. https://doi.org/10.1016/j.trc.2017.11.026.

[6] G. Wang, Z. Xu, F. Wen, K.P. Wong, Traffic-constrained multiobjective planning of electric-vehicle charging stations, IEEE Trans. Power Deliv. 28 (2013) 2363-2372.
https://doi.org/10.1109/TPWRD.2013.2269142.

[7] M.C. Catalbas, M. Yildirim, A. Gulten, H. Kurum, Estimation of optimal locations for electric vehicle charging stations, Conf. Proc. - 2017 17th IEEE Int Conf. Environ. Electr. Eng. 2017 1st IEEE Ind. Commer. Power Syst. Eur. EEEIC / I CPS Eur. 2017. (2017) https://doi.org/10.1109/EEEIC.2017.7977426

[8] V. Suresh, P. Janik, J.M. Guerrero, Z. Leonowicz, T. Sikorski, Microgrid Energy Management System with Embedded Deep Learning Forecaster and Combined Optimizer, IEEE Access. 8 (2020) 1-1. https://doi.org/10.1109/access.2020.3036131.

[9] M. Jasiński, T. Sikorski, K. Borkowski, Clustering as a tool to support the assessment of power quality in electrical power networks with distributed generation in the mining industry, Electr. Power Syst. Res. 166 (2019) 52-60. https://doi.org/10.1016/j.epsr.2018.09.020.

[10] D. Sbordone, I. Bertini, B. Di Pietra, M.C. Falvo, A. Genovese, L. Martirano, EV fast charging stations and energy storage technologies: A real implementation in the smart micro grid paradigm, Electr. Power Syst. Res. 120 (2015) 96-108 https://doi.org/10.1016/j.epsr.2014.07.033.

[11] A. Gubański, A. Sobkowiak, M. Jasiński, D. Kaczorowska, P. Janik, P. Kostyła, Z. Leonowicz, J. Rezmer, T. Sikorski, V. Suresh, Hybrid power plant with storage system: University research station, Period. Polytech. Electr. Eng. Comput. Sci. 64 (2020) 47-52. https://doi.org/10.3311/PPee.14587.

[12] A. Geron, Hands-On Machine Learing With ScikitLearn \& Tensor Flow, 2017. https://doi.org/10.3389/fninf.2014.00014.

[13] Gomes, I.; Melicio, R.; Mendes, V. Comparison between Inflexible and Flexible Charging of Electric Vehicles-A Study from the Perspective of an Aggregator. Energies 2020, 13, 5443. https://doi.org/10.3390/en13205443 